\title{
Fetal radiation dose in three common CT examinations during pregnancy Monte Carlo study
}

Kelaranta, A.

$2017-11$

Kelaranta , A , Mäkelä , T , Kaasalainen , T \& Kortesniemi , M 2017 , ' Fetal radiation dose in three common CT examinations during pregnancy Monte Carlo study ' , Physica Medica , vol. 43 , pp. 199-206 . https://doi.org/10.1016/j.ejmp.2017.09.120

http://hdl.handle.net/10138/303922

https://doi.org/10.1016/j.ejmp.2017.09.120

publishedVersion

Downloaded from Helda, University of Helsinki institutional repository.

This is an electronic reprint of the original article.

This reprint may differ from the original in pagination and typographic detail.

Please cite the original version. 
Technical note

\title{
Fetal radiation dose in three common CT examinations during pregnancy - Monte Carlo study
}

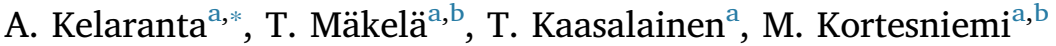 \\ ${ }^{\text {a }}$ HUS Medical Imaging Center, University of Helsinki and Helsinki University Hospital, P.O. Box 340, FI-00290 Helsinki, Finland \\ b Department of Physics, University of Helsinki, P.O. Box 64, Helsinki FI-00014, Finland
}

\section{A R T I C L E I N F O}

\section{Keywords:}

Fetal dose

Computed tomography

Monte Carlo simulation

Anthropomorphic phantom

Intrauterine dose

\begin{abstract}
A B S T R A C T
Purpose: To determine fetal doses in different stages of pregnancy in three common computed tomography (CT) examinations: pulmonary CT angiography, abdomino-pelvic and trauma scan with Monte Carlo (MC) simulations.

Methods: An adult female anthropomorphic phantom was scanned with a 64-slice CT using pulmonary angiography, abdomino-pelvic and trauma CT scan protocols. Three different sized gelatin boluses placed on the phantom's abdomen simulated different stages of pregnancy. Intrauterine dose was used as a surrogate to a dose absorbed to the fetus. MC simulations were performed to estimate uterine doses. The simulation dose levels were calibrated with volumetric CT dose index $\left(\mathrm{CTDI}_{\mathrm{vol}}\right)$ measurements and MC simulations in a cylindrical CTDI body phantom and compared with ten point doses measured with metal-oxide-semiconductor field-effect-transistor dosimeters. Intrauterine volumes and uterine walls were segmented and the respective dose volume histograms were calculated.

Results: The mean intrauterine doses in different stages of pregnancy varied from 0.04 to $1.04 \mathrm{mGy}$, from 4.8 to $5.8 \mathrm{mGy}$, and from 9.8 to $12.6 \mathrm{mGy}$ in the CT scans for pulmonary angiography, abdomino-pelvic and trauma CT scans, respectively. MC simulations showed good correlation with the MOSFET measurement at the measured locations.

Conclusions: The three studied examinations provided highly varying fetal doses increasing from sub-mGy level in pulmonary CT angiography to notably higher levels in abdomino-pelvic and trauma scans where the fetus is in the primary exposure range. Volumetric dose distribution offered by MC simulations in an appropriate anthropomorphic phantom provides a comprehensive dose assessment when applied in adjunct to point-dose measurements.
\end{abstract}

\section{Introduction}

A developing fetus has a high sensitivity to ionizing radiation $[1,2]$, and therefore, monitoring and limiting ionizing radiation exposure of an expecting mother bears a great importance. However, in certain situations, a computed tomography (CT) scan during pregnancy can be justified. The most common indications for CT during pregnancy are suspected pulmonary embolism, suspected appendicitis and trauma [3]. In these situations, the embryo or fetus can be fully or partially in the primary X-ray beam or in its immediate vicinity. It is desirable to know the absolute fetal dose in order to assess the subsequent cancer risk to the unborn child prior to a CT examination. This information is essential in the study justification and helpful in mitigating the mother's possible concerns about the radiation effects on the developing fetus.

The fetal dose cannot be measured directly inside the patient, although surface measurements could be made in vivo. However, the surface measurements do not give extensive information about the actual dose distribution inside the patient. The fetal dose can be estimated, for example, by phantom measurements with different types of dosimeters or by Monte Carlo (MC) simulations. Both methods require a model of a pregnant patient, representing the studied pregnancy stage. For that purpose, a commercially available female phantom can be modified to represent a pregnant woman. Furthermore, a fetal volume has to be defined for the corresponding anthropomorphic pregnancy model in order to determine the fetal dose. In experimental dose measurements, it is possible to obtain only discrete point doses whereas MC simulations provide a full 3D dose distribution. On the other hand, the fidelity of the MC is dependent on the assumptions and approximations in the simulation parameters and the underlying interaction model.

\footnotetext{
* Corresponding author

E-mail address: anna.kelaranta@alumni.helsinki.fi (A. Kelaranta).
} 
The uterine volume increases remarkably during pregnancy, and a large variation between individuals exists. Some studies have investigated the growth of the uterine volume and the uterine wall thickness during pregnancy [4-6], but these have tended to focus only on certain pregnancy stages and not to cover the whole pregnancy. The ICRP Publication 89 [7] provides reference values for the increase in mass of the fetus, placenta, amniotic fluid and uterus at 10,20, 30 and 38 weeks of pregnancy along with the mass of a pregravid uterus and the corresponding densities.

Some previous studies have concentrated on using MC simulations to determine fetal doses in radiological examinations [8-13]. The MC programs or codes used in these previous studies were SIERRA [8], WinODS [9], MCNPX [10,13], MCNP [11], ImpactMC [12] and EGSnrc [14]. In these studies, the stages of pregnancy ranged from early gestation ( 7 weeks) to late gestation ( 37 weeks), focusing usually on certain pregnancy stages. Most of these publications focused on CT examinations of the chest, abdomen and pelvis. A MC based method utilizing stylized mathematical phantoms to estimate the fetal dose in all stages of gestation and from any multi-detector CT examination of the trunk has also been developed [11]. Some of the very recent publications have concentrated on comparing different methods for estimating radiation dose to the conceptus [14] and comparing dosimetric and MC calculation results [15]. It has also been noticed that there is a lack of knowledge of fetal dosimetry in the imaging of pregnant women, which may lead to underestimation or overestimation of radiation dose and cancer risk [16-18]. The previous studies reporting the dose to the fetus have used either dose measurements or MC simulations, but there are few that combine them both.

The aim of this study was to determine absorbed fetal doses in different pregnancy stages (12, 20, 28 and 38 weeks) by using MC simulations and MOSFET measurements in order to validate the MC package used for the phantom geometries of this study. This approach was selected in order to achieve a comprehensive dose assessment with the volumetric dose distribution offered by MC simulations applied in conjunction to point-dose measurements. The MC simulation results for fetal dose were calibrated by reference pencil ionization chamber measurements and MC simulations in a cylindrical CT dose index (CTDI) body phantom. Pregnant female phantom geometries were achieved by modifying a physical anthropomorphic phantom. Three most common CT examinations during pregnancy were studied: pulmonary angiography, abdomino-pelvic and trauma. The intrauterine dose was used as a surrogate to a dose absorbed to the fetus.

\section{Materials and methods}

\subsection{Dose measurements}

The dose measurements in an anthropomorphic adult female phantom (CIRS ATOM 702-D, Norfolk, USA) were performed in a previous study with metal-oxide-semiconductor field-effect transistor (MOSFET) dosimeters [19]. A 64-MDCT GE LightSpeed VCT scanner (GE Healthcare, Milwaukee, WI, USA) was used in this study. Ten MOSFET dosimeters were used and dose values were registered using a mobileMOSFET 2.4.1. Dose Verification System (TN-RD-70-W, Best Medical, Canada). CT protocols for the four pregnancy stages were trauma, low-dose abdomino-pelvic and pulmonary angiography. The scan parameters are shown in Table 1 . The pitch and detector collimation were set to 1.375 and $64 \times 0.625 \mathrm{~mm}$, respectively. ASiR (adaptive statistical iterative reconstruction) level of $30 \%$ and a dose reduction option of $30 \%$ were used. In all three scan protocols, the tube current modulation (TCM) settings were according to clinical patient scans and the scan field of view (SFOV) was adjusted for a large body. The trauma CT scans resampled from $0.625 \mathrm{~mm}$ to $2.5 \mathrm{~mm}$ slice thickness were used as the MC geometries. Slice resampling was done in order to enable the simulations with existing computer memory capacity.
Table 1

The scan parameters used in the three CT protocols for the anthropomorphic phantom in the four studied pregnancy stages: 12, 20, 28 and 38 weeks. Volumetric CT dose index $\left(\mathrm{CTDI}_{\mathrm{vol}}\right)$ and dose length product (DLP) values were reported by the scanner.

\begin{tabular}{llll}
\hline & Trauma & Abdomino-pelvic & $\begin{array}{l}\text { Pulmonary } \\
\text { angiography }\end{array}$ \\
\hline Tube voltage (kVp) & 120 & 120 & 100 \\
Rotation time (s) & 0.5 & 0.5 & 0.4 \\
Scan length (mm) & 940 & 324 & 272 \\
Noise index (HU) & 30 & 42 & 38 \\
CTDI vol $_{\text {(mGy) at }}$ & $4.74 / 5.15 /$ & $2.63 / 3.04 / 3.22 /$ & $1.34 / 1.46 / 1.54 /$ \\
pregnancy weeks & $5.30 / 5.79$ & 3.91 & 1.97 \\
$\quad 12 / 20 / 28 / 38$ & & & \\
DLP (mGy.cm) at & $476.63 /$ & $102.34 / 118.04 /$ & $45.18 / 49.05 /$ \\
$\quad$ pregnancy weeks & $517.10 /$ & $127.97 / 151.86$ & $51.77 / 66.52$ \\
$12 / 20 / 28 / 38$ & $532.94 /$ & & \\
& 582.22 & & \\
& & &
\end{tabular}

In order to obtain absolute dose values, calibration measurements were performed for the ten MOSFETs using a pencil ionization chamber (RaySafe Xi, Unfors RaySafe AB, Billdal, Sweden) as the reference dosimeter. This was done as in-air measurements at the scanner isocenter for both tube voltages (100 and $120 \mathrm{kVp}$ ) used in this study.

The method used to measure beam dose-profile across the axial scan plane to determine bowtie filter thickness across the axial scan plane for the large body SFOV corresponds to previously published static X-ray tube method [20]. The dose values were measured with pencil ionization chamber at $2 \mathrm{~cm}$ intervals in air to acquire the dose profile across the beam in horizontal direction, with the tube fixed in the upright position of the gantry. The bowtie filter thicknesses were calculated based on the linear attenuation coefficients corresponding to the mean photon energy and the density of Aluminium (Al).

The accuracy of the bowtie model used in this study was verified by comparing pencil ionization chamber measurements and MC simulation results in the center and periphery positions of a cylindrical CTDI body phantom. The scanning parameters in the measurements were set according to the annual CTDI measurements protocol, and the same parameters were used in the MC simulations, including bowtie thickness data and simulated X-ray spectrum (Spekcalc 2.0, see Section 2.3) which corresponds to the methodology in previous publication [20]. The MC simulations were performed by using CT data of the body phantom, and the CTDI calculation option in the MC simulation program.

\subsection{Organ segmentation}

Intrauterine volumes and uterine walls were segmented from the CT images of the female phantom in different pregnancy stages. The absolute volumes were derived from the values presented in the ICRP Publication 89 [7]. The intrauterine volumes included fetus, placenta and amniotic fluid. The remaining exterior part of the volume was designated as uterine wall. The total uterine volume is the intrauterine volume and the uterine wall volume. This approach was selected in order to examine the deviation of the doses the slight volume change of the uterus and in order to evaluate the effect of uncertainty of the uterine wall volume variability which can be taken into account in the total uncertainty evaluation. The pregnancy stages and the corresponding intrauterine volumes and uterine wall volumes are shown in Table 2. According to the phantom manufacturer, the phantom was made of tissue-equivalent epoxy resins with the following densities: bone tissue $\left(1.60 \mathrm{~g} / \mathrm{cm}^{3}\right)$, soft tissue $\left(1.05 \mathrm{~g} / \mathrm{cm}^{3}\right)$, spinal cord $(1.07 \mathrm{~g}$ / $\left.\mathrm{cm}^{3}\right)$, spinal disks $\left(1.15 \mathrm{~g} / \mathrm{cm}^{3}\right)$ and lungs $\left(0.21 \mathrm{~g} / \mathrm{cm}^{3}\right)$. The different stages of pregnancy were constructed with home-made gelatin boluses by mixing water and gelatin in a heat bath and casting a bolus in a spherical mold with varying spherical segment volumes for 20, 28 and 38 weeks of pregnancy. At 12 weeks of pregnancy, no bolus was used. 
Table 2

The pregnancy stages and the corresponding uterine wall and intrauterine volumes.

\begin{tabular}{lll}
\hline Pregnancy stage (weeks) & Intrauterine volume $(\mathrm{ml})$ & Uterine wall volume (ml) \\
\hline 12 & $210^{\dagger}$ & $240^{\dagger}$ \\
20 & 840 & 380 \\
28 & $2300^{\dagger}$ & $590^{\dagger}$ \\
38 & 4800 & 1000 \\
\hline
\end{tabular}

${ }^{\dagger}$ Linearly interpolated from the values reported in the ICRP publication 89 for 10, 20, 30 and 38 weeks [7].

The gelatin boluses had a contrast of $7 \pm 22 \mathrm{HU}$ (average \pm 1 standard deviation, SD), according to the $120 \mathrm{kVp}$ scan. The bolus sizes and uterine wall segmentations for pregnancy weeks 12, 20, 28 and 38 are presented in Fig. 1. The bolus volumes were 6.5, 18.0, and $40.6 \mathrm{dl}$ for the pregnancy stages of 20,28 and 38 weeks, respectively. Phantom circumferences were approximately $77 \mathrm{~cm}, 83 \mathrm{~cm}, 88 \mathrm{~cm}$ and $94 \mathrm{~cm}$ for 12, 20, 28 and 38 weeks, respectively. These were measured just above the ilium where the boluses' cross sections were at their largest. Phantom's waist was $69 \mathrm{~cm}$ at minimum. The delineations were adjusted to fulfill the ICRP references volumes and to follow the anthropomorphic phantom geometry, including the pregnancy stage specific bolus volume and shape. The intrauterine dose was used as a surrogate to a dose absorbed to the fetus.

\subsection{Dose simulations}

ImpactMC software (GPU version 1.4.0.0, 2014, ImpactMC, Vamp $\mathrm{GmbH}$, Germany) was used for the MC dose simulations. The program
Table 3

MC parameters in ImpactMC

\begin{tabular}{|c|c|}
\hline Property & Value \\
\hline Focus to center of rotation distance (mm) & 541 \\
\hline Fan angle (rad) & 0.9 \\
\hline Number of rotations ${ }^{1 / 2 / 3}$ & $5 / 6 / 17$ \\
\hline Rotation time $(\mathrm{s})^{1 / 2 / 3}$ & $0.4 / 0.5 / 0.5$ \\
\hline Start angle (rad) & 0.0 \\
\hline Table increment (mm) & 55 \\
\hline Total beam collimation (mm) & 40 \\
\hline $\mathrm{X}$-ray tube voltage $(\mathrm{kVp})$ & $100 / 120 / 120$ \\
\hline Break energy $(\mathrm{keV})$ & 10.0 \\
\hline Maximum number of photons & $5 \mathrm{E}+09$ \\
\hline Number of interactions & 10 \\
\hline Number of projections per rotation ${ }^{1 / 2 / 3}$ & $87 / 86 / 89$ \\
\hline
\end{tabular}

\footnotetext{
${ }^{1}$ Pulmonary angiography.

2 Abdomino-pelvic.

${ }^{3}$ Trauma.
}

allows an adaptation to scanner-specific irradiation geometries and individual patient or phantom characteristics. Prior to the MC simulation, the X-ray spectra applied in the CT scans were simulated by using information from the vendor specifications (tube type, half value layer, anode material and filtration) and Spekcalc 2.0 program [21]. The bowtie thickness data and simulated X-ray spectrum were then used as an input for the MC simulations, together with the anthropomorphic phantom CT voxel data, CT examination specific longitudinal scan ranges and tube current values based on the data. The TCM accounted for tube current variations in $\mathrm{z}$-axis direction, and values were collected from the DICOM headers of the phantom CT slices in the three scan

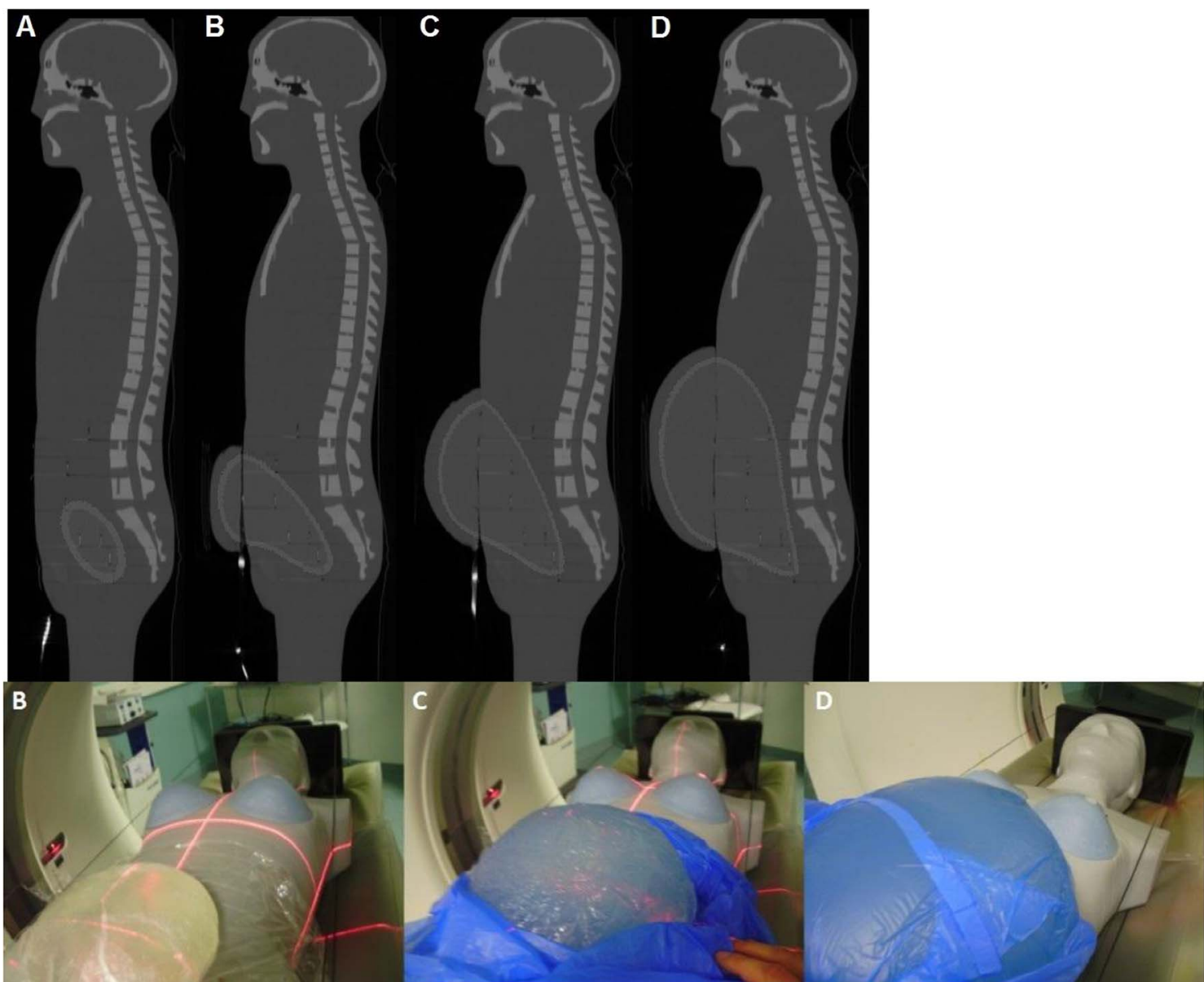

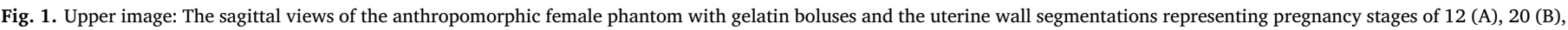
28 (C) and 38 (D) weeks. Lower image: The phantom and the gelatin boluses at pregnancy stages of 20 (B), 28 (C) and 38 (D) weeks. 
protocols. Furthermore, symmetric collimation, positive rotation direction and spiral scanning mode was used in the simulations. The main MC simulation parameters are shown in Table 3. The time per simulation was approximately $1.5 \mathrm{~h}$ for the used maximum number of photons, 5E +09 .

In this study, MC simulation results for fetal dose were calibrated by pencil ionization chamber measurements and MC simulations in a cylindrical CTDI body phantom. Mean MC doses were recorded at MOSFET positions using 15-mm-diameter spherical volumes-of-interest (VOIs). The VOI size was greater than the dosimeter active volume and was selected to ensure adequate statistics and to avoid confounding effects from air and metal at the dosimeter sites. The voxels containing metal were excluded from the dose VOI. The MC results were scaled according to the $\mathrm{CTDI}_{\mathrm{vol}-\mathrm{meas}} / \mathrm{CTDI}_{\mathrm{vol}-\mathrm{MC}}$ ratio to obtain the final simulated dose maps. The MC simulation results and MOSFET measurement results were compared for more comprehensive dosimetric evaluation. An absolute dose distribution was acquired from each simulation. Linear fit with forced intercept at the origin was used in the comparison between the MC simulation and MOSFET measurement points.

Absorbed doses to the intrauterine volumes and uterine walls were determined by co-registering the 3D dose maps with the segmented volumes. Integral dose volume histograms, dose distribution quartile values and mean values were determined from each pregnancy stage, from each scan protocol and for both intrauterine volume and uterine wall volume. Source CT image data which was used in the simulations included local inhomogeneities due to small air cavities on the bolusphantom interface and due to MOSFET dosimeter structures. The corresponding simulated voxel dose values were not regarded as representative of the actual tissue dose and therefore those voxel doses were omitted in the overall volumetric dose determination.

\section{Results}

\subsection{Dose measurements and simulations}

CTDI body phantom MC simulations resulted in $8.5 \%$ and $6.1 \%$ lower $\mathrm{CTDI}_{\mathrm{vol}}$-values compared to pencil chamber dosimeter measurements when $120 \mathrm{kVp}$ and $100 \mathrm{kVp}$ tube voltages, respectively, $40 \mathrm{~mm}$ collimation and large SFOV were used. The $\mathrm{CTDI}_{\text {vol-meas }} / \mathrm{CTDI}_{\mathrm{vol}-\mathrm{MC}}$ ratios were 1.093 and 1.065 for $120 \mathrm{kVp}$ and $100 \mathrm{kVp}$, respectively. $\mathrm{CTDI}_{\mathrm{vol}}$ displayed by the scanner console divided by the measured air kerma at the isocenter were 0.36 and 0.33 for $120 \mathrm{kVp}$ and $100 \mathrm{kVp}$, respectively, $40 \mathrm{~mm}$ total beam collimation (projected to scan isocenter) and large SFOV.

MC dose map scaling factors obtained from linear fits with MOSFET measurements ranged from 0.99 to 1.05 for trauma, from 0.91 to 1.04 for abdomino-pelvic and from 0.44 to 0.52 for pulmonary angiography protocols. The results for trauma, abdomino-pelvic and pulmonary angiography protocols are shown in Fig. 2 with linear regression lines fitted to all MOSFET measurement points inside the phantom, with the corresponding scaling factors and coefficients of determination, $\mathrm{R}^{2}$.

\subsection{Uterine doses}

Tables 4-6 present the simulated and measured mean doses in different pregnancy stages in pulmonary angiography, abdomino-pelvic and trauma CT scan protocols, respectively. Integral dose volume histograms corresponding to the studied pregnancy stages and scan protocols are presented in Fig. 3. Box-and-whisker plots showing the 2nd and 98th percentiles, the first and third quartiles and median dose levels for each pregnancy stage and scan protocol are shown in Fig. 4. Absorbed doses to the intrauterine volumes were the greatest in the trauma CT scan, whereas the intrauterine doses were lowest in the pulmonary CT angiography scan.

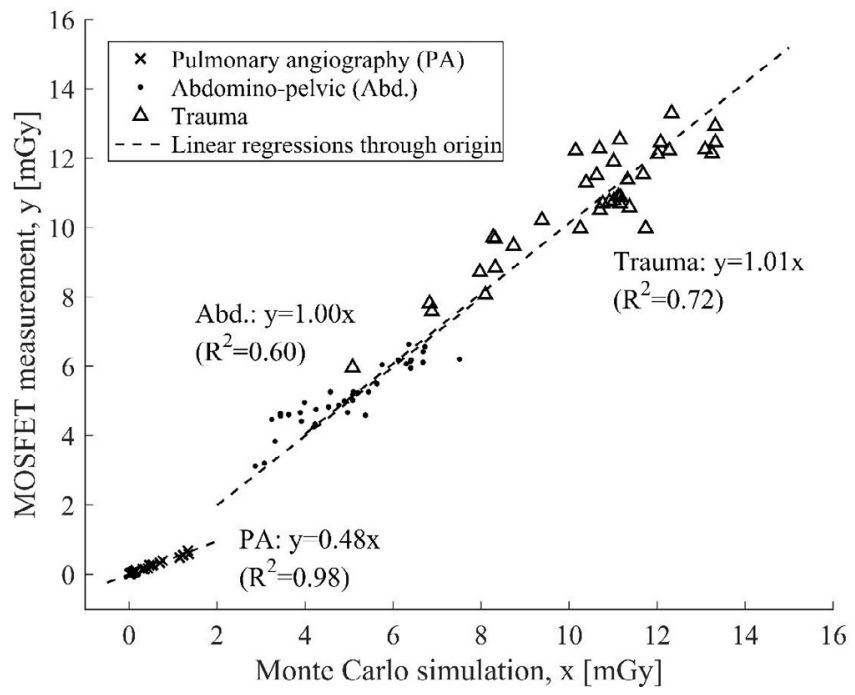

Fig. 2. Absorbed dose at MOSFET dosimeter positions in trauma abdomino-pelvic (Abd.) and pulmonary angiography (PA) protocols as a function of the MC simulations results at the same positions.

Table 4

The pregnancy stages and the corresponding simulated mean doses to the intrauterine volumes and total uterine volumes in the pulmonary angiography CT scan protocol. Corresponding MOSFET measurement results are shown for comparison.

\begin{tabular}{llll}
\hline $\begin{array}{l}\text { Pregnancy stage } \\
\text { (weeks) }\end{array}$ & $\begin{array}{l}\text { Intrauterine dose } \\
\text { (mGy) }\end{array}$ & $\begin{array}{l}\text { Total uterine } \\
\text { dose (mGy) }\end{array}$ & $\begin{array}{l}\text { Fetal dose in MOSFET } \\
\text { measurements (mGy) } \\
{[19]}\end{array}$ \\
\hline 12 & 0.04 & 0.04 & 0.03 \\
20 & 0.09 & 0.09 & 0.08 \\
28 & 0.27 & 0.29 & 0.14 \\
38 & 1.04 & 1.13 & 0.22 \\
\hline
\end{tabular}

Table 5

The pregnancy stages and the corresponding simulated mean doses to the intrauterine volumes and total uterine volumes in the low-dose abdomino-pelvic CT scan protocol. Corresponding MOSFET measurement results are shown for comparison.

\begin{tabular}{llll}
\hline $\begin{array}{l}\text { Pregnancy stage } \\
\text { (weeks) }\end{array}$ & $\begin{array}{l}\text { Intrauterine dose } \\
\text { (mGy) }\end{array}$ & $\begin{array}{l}\text { Total uterine } \\
\text { dose (mGy) }\end{array}$ & $\begin{array}{l}\text { Fetal dose in MOSFET } \\
\text { measurements (mGy) } \\
{[19]}\end{array}$ \\
\hline 12 & 5.2 & 5.1 & 4.7 \\
20 & 5.8 & 5.8 & 5.5 \\
28 & 4.9 & 4.9 & 4.9 \\
38 & 4.8 & 4.8 & 5.1 \\
\hline
\end{tabular}

Table 6

The pregnancy stages and the corresponding simulated mean doses to the intrauterine volumes and total uterine volumes in the trauma CT scan protocol. Corresponding MOSFET measurement results are shown for comparison.

\begin{tabular}{llll}
\hline $\begin{array}{l}\text { Pregnancy stage } \\
\text { (weeks) }\end{array}$ & $\begin{array}{l}\text { Intrauterine dose } \\
\text { (mGy) }\end{array}$ & $\begin{array}{l}\text { Total uterine } \\
\text { dose (mGy) }\end{array}$ & $\begin{array}{l}\text { Fetal dose in MOSFET } \\
\text { measurements (mGy) } \\
{[19]}\end{array}$ \\
\hline 12 & 11.3 & 11.3 & 10.6 \\
20 & 12.6 & 12.6 & 11.2 \\
28 & 10.3 & 10.3 & 10.1 \\
38 & 9.8 & 9.9 & 10.7 \\
\hline
\end{tabular}

\subsection{Uncertainty calculations}

In the MC simulations, the relative statistical uncertainty of voxel dose $(\mathrm{SD} /$ mean) in uterine region was approximately $10 \%$ in the abdominal and trauma scans, and considerably higher $(>100 \%)$ in the 

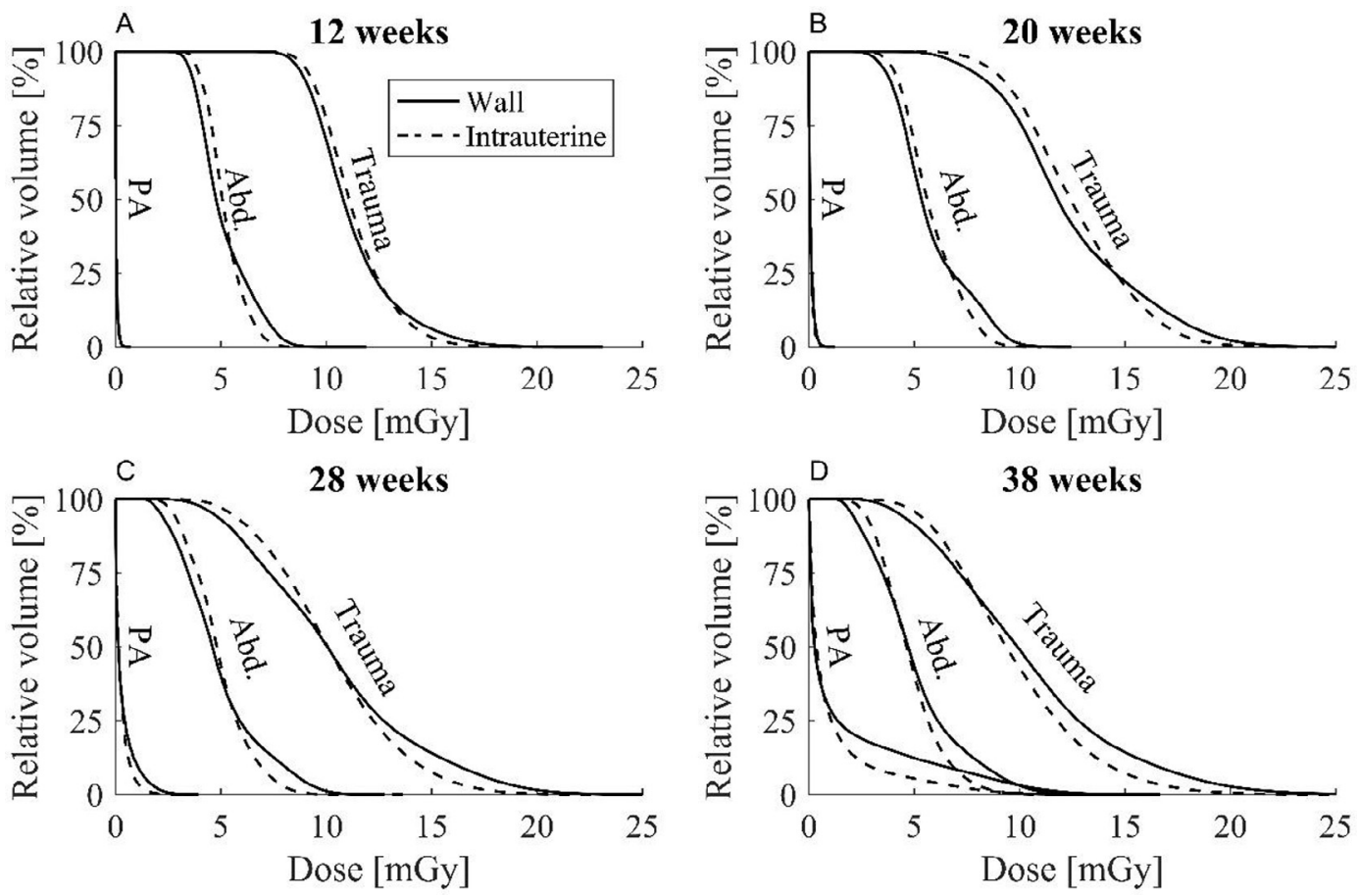

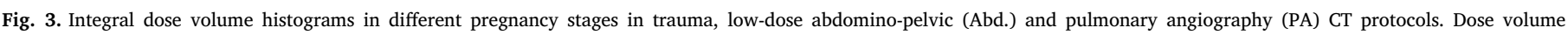
histograms were determined for both intrauterine volume (dashed line) and uterine wall volume (solid line).

pulmonary angiography scans where uterus volume is out of the primary scan region and receives only scattered radiation. However, the uterus or any practical (macroscopic) volume of interest contain very large amount of voxels. Therefore, according to the standard error of the mean, a reasonable upper estimate of the statistical uncertainty of the mean dose to such volume is $1 \%$, providing only minor contribution to the overall uncertainty.

The estimated overall uncertainty of the fetal dose was $30 \%$ with the fetal segmentation uncertainty, and $15 \%$ without the fetal segmentation uncertainty. The relative dosimetric effect of the fetal segmentation uncertainty is based on the variability of the dose distribution within the uterine volume which is demonstrated by the interquartile dose ranges in the box-and-whisker plots of the Fig. 4. This provides the reasonable boundaries for the use of uterine dose as the surrogate of the fetal dose. The relative differences in the fetal dose uncertainty due to segmentation were higher in the pulmonary angiography scan where the scattered radiation is directed caudally towards the fetus from the chest volume of the mother. In the abdomino-pelvic and trauma scans, the relative difference between mean intrauterine dose and third quartile intrauterine dose was smaller in the early pregnancy (about 10\%) and increased towards the late pregnancy (about $20-25 \%$ ). General estimated uncertainty of $25 \%$ from the fetal

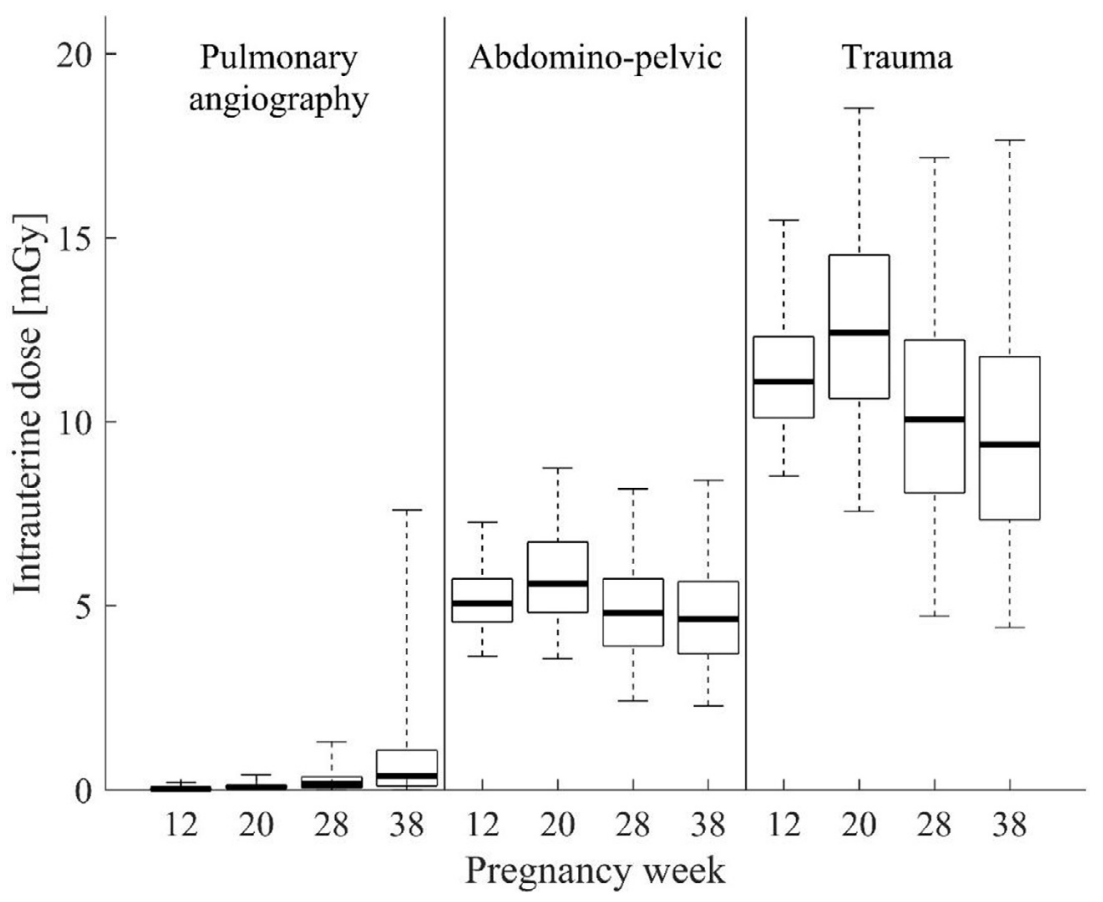

Fig. 4. Box-and-whisker plots presenting the 2nd and 98th percentile, the first and third quartiles and median values of the intrauterine dose distributions in the four modelled pregnancy stages for the three CT protocols. 
dose due to segmentation effect was used in the net uncertainty evaluation. The other uncertainty factors included spectral and bowtie modeling uncertainties [20], CTDI-calibration uncertainty [22,23], modeling of the mA-modulation and tube start angle, and MC simulation statistical uncertainty with the used photon counts (see Section 4), providing a combined $15 \%$ uncertainty.

\section{Discussion}

CT imaging of a pregnant woman is sometimes necessary due to potential and serious health risks either to the mother or the unborn child. The pregnancy might also be unknown at the time of the examination. The most common indications for the CT examination during pregnancy are suspected pulmonary embolism, suspected appendicitis, and trauma [3]. These three indications are also covered in a recent review article about the clinical perspective on diagnostic X-ray examinations of pregnant patients [24]. When the scan is appropriately justified and needed, the benefits of the CT scan outweigh the small radiation risk to the fetus. The radiation exposure to the fetus depends significantly, among other factors, on the scan range and irradiation geometry, and the fetus's position relative to it. The exact position of the fetus in the uterus vary and is unknown for the prospective dose estimation. Therefore, the dose absorbed by the uterus can serve as a surrogate for the dose absorbed by the embryo or fetus in medical radiation dosimetry. In addition to these assumptions, patient specific TCM can cause differences in the absorbed doses even when the model and the patient match roughly in size [14]. There are also differences between TCM techniques used by different vendors. The aim of this study was to determine absorbed fetal doses for the most common CT indications during pregnancy by using MC simulations and MOSFET measurements in order to validate the MC package used for the phantom geometries of this study. For this purpose, MC simulations were performed for an anthropomorphic female phantom containing different sized spherical segment boluses on the phantom's abdomen to simulate different stages of pregnancy. The results of this study strengthen the knowledge about fetal doses in CT examinations, and fetal dose comparisons that can be done between CT scanners from different vendors are also important.

The simulated mean intrauterine doses in different stages of pregnancy varied from 0.04 to $1.04 \mathrm{mGy}$, from 4.8 to $5.8 \mathrm{mGy}$, and from 9.8 to $12.6 \mathrm{mGy}$ in the CT scans for pulmonary angiography, abdominopelvic and trauma CT scans, respectively. These values are in a good correspondence with the previously published fetal doses that were based on MOSFET measurements [19]. The small differences can be caused by possible imperfections in the Monte Carlo parameters, such as the energy spectra and the bowtie filter model. Other possible inaccuracies can results from the fact that not all parameters affecting the MC simulations are available from the manufacturer or they cannot be known exactly, such as the central bowtie filter thickness or the starting position of the X-ray tube. Forced intercept at the origin was chosen by the assumption that both methods should agree at zero dose. The method was considered to be robust when e.g. the unknown X-ray tube starting position could affect the dosimeter point-dose values and result in erroneous biases. The fitting functions were visually verified to agree well with the data points. MOSFET dose values for the pulmonary angiography protocol were a reverse from the other two protocols: approximately half of the simulated values (see Fig. 2). However, according to the values in Table 4, the difference between the mean simulated doses to the intrauterine volumes and the fetal doses in MOSFET measurements varies for different pregnancy stages. This is due to the fact that certain sets of MOSFETs were chosen to represent the fetal volume in different pregnancy stages, and this choice cannot equally represent the $3 \mathrm{D}$ dose distribution and the resulting mean simulated dose to the intrauterine volume. The correlation between doses at single MOSFET positions and MC simulation results at the same position was good. Moreover, the angular dependency of the MOSFETs causes lower sensitivity at the distal tip and stem direction [25], and in the pulmonary angiography protocol, irradiation from this direction had a larger contribution to the total dose than in the trauma and pulmonary angiography protocols. Nevertheless, the dose level in the pulmonary CT angiography scan was very low. The correction factors for trauma and abdominal protocols matched well with the CTDI body phantom simulation vs. measurement results.

Many previous studies focus only on measurements or on simulations, but there are few that combine them both $[15,26,27]$, as was done in this study. MC simulations can provide 3D dose distributions, which are not possible with a discrete number of physical dosimeters. However, the measurements and simulations should match, since the used parameters were set to be as close to each other as possible. The advantage of this approach is that the point dose measurement results can be compared with the simulation results in order to give comprehensive results of the dose distributions. The agreement between the CTDI measurements and CTDI simulations were in conformance with previous MC program validations [22,23]. The results of this study also compare reasonably well with previously published fetal dose values [8-15]. Deviations were expected due to TCM and differences in the scanning parameters and phantom properties. Especially the doses from pulmonary angiography and trauma CT were found to be close to the previously published results $[8,9]$. A previous study that utilized ImpactMC simulations in abdomino-pelvic CT examinations in early pregnancy showed fetal doses of 0.34 to $0.79 \mathrm{mGy}$ at $120 \mathrm{kVp}$ when normalized to absorbed dose in air [12]. The corresponding value of the present study at 12 weeks of pregnancy was $0.64 \mathrm{mGy}$, which is in good agreement with the previous study. If the CT examination is performed with a fixed tube current $[10,11]$, the results are not directly comparable with CT examinations performed with TCM. Moreover, the volume representing the fetus may be defined differently. If the fetus is segmented and the absorbed dose to the fetal tissues is determined, it is likely to differ from the dose to the intrauterine volume, especially if the dose distribution is inhomogeneous. It has been found that the fetal doses in late pregnancy are higher than in early and mid-pregnancy when performing abdomino-pelvic CT scans with TCM [13], whereas in this study, the fetal dose remained fairly constant in all stages of pregnancy in the abdomino-pelvic and trauma CT scans due to the automatic TCM used. In this study, the higher doses at 20 weeks can be explained by tube current modulation, phantom geometry and the shape of the uterus, its position and location.

The variability range in the absorbed dose to the intrauterine volume was large, as can be seen in Figs. 3 and 4. The high variations in the intrauterine doses in the trauma and abdomino-pelvic CT scans were primarily caused by the rotating tube in helical imaging resulting in "zebra" lines to the dose maps (Fig. 5A). The distance of the fetus from the scan range had an effect on the fetal dose as observed in the pulmonary angiography CT scan. According to the dose volume histograms, the fetal dose in the pulmonary angiography CT scan was negligible in the early pregnancy but started to increase as the intrauterine volume increased and the distance from the scanning range decreased. In abdomino-pelvic and trauma CT scans, the uterus was in the primary scanning range, and therefore the intrauterine dose was significantly higher and more consistent compared to radiation exposure to intrauterine volume in pulmonary angiography CT scan.

The uterus was segmented based on reference volumes and the chosen bolus shapes while the inferior edge was fixed. This choice did not represent any individual human subject and different segmentations would have resulted in slightly different mean absorbed doses. The uncertainty contribution related to the uterine segmentation on the dose determination can be seen from the box-and-whisker plots in the Fig. 4 and by comparing the intrauterine doses to the combined intrauterine and uterine wall doses. The total mean uterine doses (intrauterine volume and uterine wall volume combined) in the pulmonary CT angiography scans were 5\%,3\%, 5\% and 9\% higher than the mean intrauterine doses for the pregnancy stages 12, 20, 28 and 

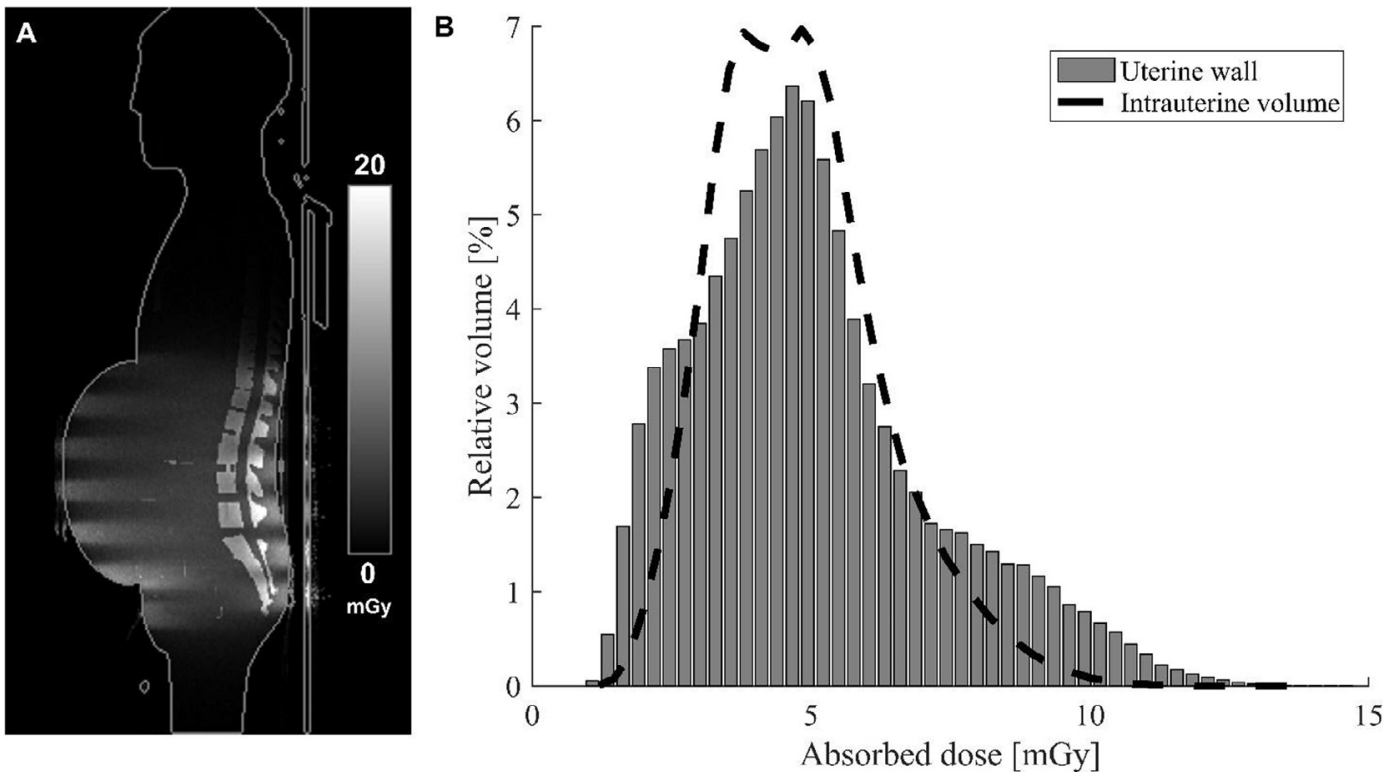

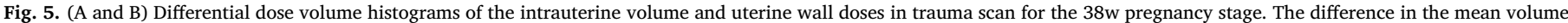
dose was $6 \%$.

38 weeks, respectively. In contrast, the dose remained more constant, within one percent, in the abdomino-pelvic and trauma CT scan protocols. Differences were larger when comparing the intrauterine doses to the uterine wall doses. The differential dose volume histograms of the intrauterine volume and uterine wall doses in abdomino-pelvic scan for the 38 weeks pregnancy stage is presented in Fig. 5B to exemplify differences in the overall dose distributions. An upper bound of the fetal doses in the simulated cases and chosen segmentations could be estimated by investigating the mean dose in the high end of the dose histograms. Assuming that the fetus accounts for 9\% (12 weeks), 37\% (20 weeks), 56\% (28 weeks) and 69\% (38 weeks) of the intrauterine volumes [7], the maximum theoretical fetal doses would then have been 15.5, 7.5 and $0.8 \mathrm{mGy}$, for trauma, abdominal and pulmonary angiography protocol, respectively.

There are limitations in this study. The intrauterine dose was assumed to simulate the radiation dose absorbed to the fetus. However, the intrauterine dose does not represent the fetal dose exactly because the size and position of the fetus depends on the gestational age and varies between individuals. Moreover, the uncertainties of the fetal dose have several aspects: in early pregnancy, the embryo's or fetus's cells will likely be exposed to a relatively uniform dose, but the location of fetus in the uterus may not be known; in later stages of pregnancy, the position of the fetus in the uterus may vary, and different organs and tissues may no longer be exposed to the same doses. Furthermore, the uterine segmentation and alignment modeling in this study was in part guided by the physical boluses which might not be representative for all clinical cases. Finally, in this study dose simulations were applied to a single phantom model, for one scanner model and clinical protocol.

\section{Conclusions}

Volumetric dose distribution offered by MC simulations compared with experimental point-dose measurements provides a comprehensive dose assessment of the patient model including variations in different scan protocols and pregnancy stages. MC simulations showed good correlation with the MOSFET measurement at the measured locations. The fetal doses were highest in the trauma CT protocol due to the longer scan range and higher exposure parameters. The pregnancy stage did not have a significant effect on the magnitude of the fetal doses in the trauma or abdomino-pelvic scans. In the pulmonary angiography scan, the fetal doses increased with pregnancy stage as the fetus was closer to the CT scan range in the later pregnancy. However, the pulmonary CT angiography scan represented sub-mGy fetal dose range irrespective of the pregnancy stage. As a proper reminder for the concerned mothers, the radiation exposure to the fetus resulting from any of the studied CT scans should not be considered as a reason to terminate the pregnancy.

\section{Acknowledgements}

The authors are grateful to the Radiation and Nuclear Safety Authority of Finland (STUK) for the possibility to use the ImpactMC software.

The authors are grateful to radiologist Raija Seuri for evaluating the segmented uterus volumes.

\section{References}

[1] ICRP (International Commission on Radiological Protection). ICRP publication 90. Biological Effects after prenatal irradiation (embryo and fetus). Ann ICRP 2003;33(1-2).

[2] ICRP (International Commission on Radiological Protection). ICRP publication 103. The 2007 recommendations of the international commission on radiological protection. Ann ICRP 2007;37(2-3).

[3] Goldberg-Stein S, Liu B, Hahn PF, Lee SI. Body CT during pregnancy: utilization trends, examination indications, and fetal radiation doses. Am J Roentgenol 2011;196:146-51. http://dx.doi.org/10.2214/AJR.10.4271.

[4] Geirsson RT, Ogston SA, Patel NB, Christie AD. Growth of total intrauterine, intraamniotic and placental volume in normal singleton pregnancy measured by ultrasound. Br J Obstet Gynaecol 1985;92(1):46-53.

[5] Goldstein SR, Horii SC, Snyder JR, Raghavendra BN, Subramanyam B. Estimation of nongravid uterine volume based on a nomogram of gravid uterine volume: its value in gynecologic uterine abnormalities. Obstet Gynecol 1988;72(1):86-90.

[6] Sokolowski P, Saison F, Giles W, McGrath S, Smith D, Smith J, et al. Human uterine wall tension trajectories and the onset of parturition. PLoS One 2010;5(6):e11037. http://dx.doi.org/10.1371/journal.pone.0011037.

[7] ICRP (International Commission on Radiological Protection). ICRP publication 89. Basic anatomical and physiological data for use in radiological protection: reference values. Ann ICRP 2002;32(3-4).

[8] Winer-Muram HT, Boone JM, Brown HL, Jennings SG, Mabie WC, Lombardo GT. Pulmonary embolism in pregnant patients: fetal radiation dose with helical CT. Radiology 2002;224:487-92. http://dx.doi.org/10.1148/radiol.2242011581.

[9] Helmrot E, Pettersson H, Sandborg M, Altén JN. Estimation of dose to the unborn child at diagnostic X-ray examinations based on data registered in RIS/PACS. Eur Radiol 2007;17:205-9. http://dx.doi.org/10.1007/s00330-006-0286-2.

[10] Angel E, Wellnitz CV, Goodsitt MM, Yaghmai N, DeMarco JJ, Cagnon CH, et al. Radiation dose to the fetus for pregnant patients undergoing multidetector CT imaging: Monte Carlo simulations estimating fetal dose for a range of gestational age and patient size. Radiology 2008;249:220-7. http://dx.doi.org/10.1148/radiol. 2491071665. 
[11] Damilakis J, Tzedakis A, Perisinakis K, Papadakis AE. A method of estimating conceptus doses resulting from multidetector CT examinations during all stages of gestation. Med Phys 2010;37:6411-20. http://dx.doi.org/10.1118/1.3517187.

[12] Damilakis J, Perisinakis K, Tzedakis A, Papadakis AE, Karantanas A. Radiation dose to the conceptus from multidetector CT during early gestation: a method that allows for variations in maternal body size and conceptus position. Radiology 2010;257:483-9. http://dx.doi.org/10.1148/radiol.10092397.

[13] Gu J, George XuX, Caracappa PF, Liu B. Fetal doses to pregnant patients from ct with tube current modulation calculated using Monte Carlo simulations and realistic phantoms. Radiat Prot Dosimetry 2013;155:64-72. http://dx.doi.org/10. 1093/rpd/ncs312.

[14] Lopez-Rendon X, Walgraeve MS, Woussen S, Dedulle A, Zhang G, Bosmans H, et al. Comparing different methods for estimating radiation dose to the conceptus. Eur Radiol 2016:1-8. http://dx.doi.org/10.1007/s00330-016-4389-0.

[15] Matsunaga Y, Kawaguchi A, Kobayashi M, Suzuki S, Suzuki S, Chida K. Radiation doses for pregnant women in the late pregnancy undergoing fetal-computed tomography: a comparison of dosimetry and Monte Carlo simulations. Radiol Phys Technol 2016:1-7. http://dx.doi.org/10.1007/s12194-016-0377-y.

[16] Groves AM, Yates SJ, Win T, Kayani I, Gallagher FA, Syed R, et al. CT pulmonary angiography versus ventilation-perfusion scintigraphy in pregnancy: implications from a UK survey of doctors' knowledge of radiation exposure. Radiology 2006;240:765-70. http://dx.doi.org/10.1148/radiol.2403050910.

[17] Ramanathan S, Ryan J. Radiation awareness among radiology residents, technologists, fellows and staff: where do we stand? Insights Imaging 2015;6:133-9. http://dx.doi.org/10.1007/s13244-014-0365-x.

[18] Ratnapalan S, Bona N, Chandra K, Koren G. Physicians' perceptions of teratogenic risk associated with radiography and CT during early pregnancy. Am J Roentgenol 2004;182:1107-9. http://dx.doi.org/10.2214/ajr.182.5.1821107.

[19] Kelaranta A, Kaasalainen T, Seuri R, Toroi P, Kortesniemi M. Fetal radiation dose in computed tomography. Radiat Prot Dosimetry 2015;165:226-30. http://dx.doi. org/10.1093/rpd/ncv097.

[20] Alikhani B, Büermann L. Non-invasive experimental determination of a CT source model. Phys Med 2016;32(1):59-66. http://dx.doi.org/10.1016/j.ejmp.2015.09. 006.

[21] Poludniowski G, Landry G, DeBlois F, Evans PM, Verhaegen F. SpekCalc: a program to calculate photon spectra from tungsten anode X-ray tubes. Phys Med Biol 2009;54:N433-8. http://dx.doi.org/10.1088/0031-9155/54/19/N01.

[22] Deak P, van Straten M, Shrimpton PC, Zankl M, Kalender WA. Validation of a Monte Carlo tool for patient-specific dose simulations in multi-slice computed tomography. Eur Radiol 2008;18(4):759-72. http://dx.doi.org/10.1007/s00330-0070815-7.

[23] Myronakis M, Perisinakis K, Tzedakis A, Gourtsoyianni S, Damilakis J. Evaluation of a patient-specific Monte Carlo software for CT dosimetry. Radiat Prot Dosimetry 2009;133(4):248-55. http://dx.doi.org/10.1093/rpd/ncp051.

[24] Vock P. Clinical perspective on diagnostic X-ray examinations of pregnant patients what to take into account. Phys Med 2017. http://dx.doi.org/10.1016/j.ejmp.2017. 05.004 .

[25] Koivisto J, Kiljunen T, Wolff J, Kortesniemi M. Characterization of MOSFET dosimeter angular dependence in three rotational axes measured free-in-air and in softtissue equivalent material. J Radiat Res 2013;54(5):943-9. http://dx.doi.org/10. 1093/jrr/rrt015.

[26] Gu J, Bednarz B, Caracappa PF, Xu XG. The development, validation and application of a multi-detector CT (MDCT) scanner model for assessing organ doses to the pregnant patient and the fetus using Monte Carlo simulations. Phys Med Biol 2009;54(9):2699-717. http://dx.doi.org/10.1088/0031-9155/54/9/007.

[27] DeMarco JJ, Cagnon CH, Cody DD, Stevens DM, McCollough CH, O'Daniel J, et al. A Monte Carlo based method to estimate radiation dose from multidetector CT (MDCT): cylindrical and anthropomorphic phantoms. Phys Med Biol 2005;50(17):3989-4004. http://dx.doi.org/10.1088/0031-9155/50/17/005. 